\title{
PHYSICAL PARAMETERS OF INHOMOGENEITIES IN WOLF-RAYET WINDS
}

\author{
C. ROBERT ${ }^{1}$, A. F. J. MOFFAT ${ }^{1}$, and W. SEGGEWISS ${ }^{2}$ \\ ${ }^{1}$ Université de Montréal, Département de physique, Montréal, and Observatoire du \\ mont Mégantic, Canada \\ ${ }^{2}$ Observatorium Hoher List, Universitäts-Sternwarte Bonn, F. R. Germany
}

\begin{abstract}
Time-resolved spectroscopy has revealed small, systematically varying features superimposed on the broad emission lines of WR stars. We believe that these structures are due to inhomogeneities of emitting wind material propagating with the general wind. Here we suggest a common, but still unknown, origin for the intrinsic variations observed in WR stars.
\end{abstract}

\section{INTRODUCTION}

Photometric and polarimetric observations of Wolf-Rayet (WR) stars indicate that these objects can show intrinsic variations with time scales of hours to days. Late-type stars of both sequences, WN8 and WC9, are the most variable (e.g. Lamontagne and Moffat 1987; Robert et al. 1989). No clear periodicity can by unambiguously discerned. Therefore, we favor an explanation for these variations as due to stochastically fluctuating inhomogeneities in the winds.

In the spectra, variations in the shapes of the line profiles are also observed. Here we present new spectra of three WR stars of different spectral type: HD $96548=$ WR40 (WN8), HD $164270=$ WR103 (WC9) and HD $165763=$ WR111 (WC5). Along with the WN6 stars HD $191765=$ WR134 and HD 192163 = WR136 (McCandliss 1988; Moffat et al. 1988), we describe and compare, in a qualitative way for now, the variations in the lines.

\section{OBSERVATIONS AND ANALYSIS}

The new spectra presented here were collected from 1989 May 31 to June 2 (UT) with the echelle spectrograph CASPEC and a CCD detector at the ESO $3.6 \mathrm{~m}$ telescope, Chile. High signal-to-noise $(300-500)$ and high resolution $(0.07 \AA / \mathrm{pix})$ spectra were obtained in the range 5160 to $6190 \AA$.

Special care has been taken to eliminate the ripple appearing when merging together the different spectral orders obtained with the echelle. A routine involving Fourier analysis has been developed for this purpose.

Line variability was then assessed by comparing the standard deviation (rms difference from the mean for each night) in the line with the standard deviation in the continuum. A variable line is taken to be one for which $\sigma_{\text {line }}>3 \sigma_{\text {continuum }}$, in excess of the Poisson errors.

\section{RESULTS AND DISCUSSION}

1) The variability

In the WC5 star HD 165763 , the C IV 5805 and especially the C III 5696 lines are noticibly variable (see Fig. 1). Small variations are also seen in the He II 5411, C IV 5471 and O III 5592 lines.

Almost all the lines of the WC9 star HD 164270 are strongly variable (see Fig. 2). The HeII 5411 and O III 5592 lines show a P Cyg profile, where changes occur in both the emission and the absorption parts (He I 5876 also has a P Cyg profile but which is strongly blended).

Many lines between 3950 and $6610 \AA$ of the WN6 star HD 191765 were also found to be variable (McCandliss 1988). For the other WN6 star, HD 192163, the He II 5411 line shows less activity during the night of 1986 July 26 (UT) than the same line in HD 191765 (Moffat et al. 1988).

The WN8 star HD 96548 shows strong P Cyg lines of He II 5411 and He I 5876 which are considerably variable both in the emission and the absorption parts of the profile (Fig. 3).

A closer look at the variable lines reveals the presence of small subpeaks or bumps which move with time (e.g. C III 5696 line of HD 165763, Fig. 1). These bumps have a short life-time of the 


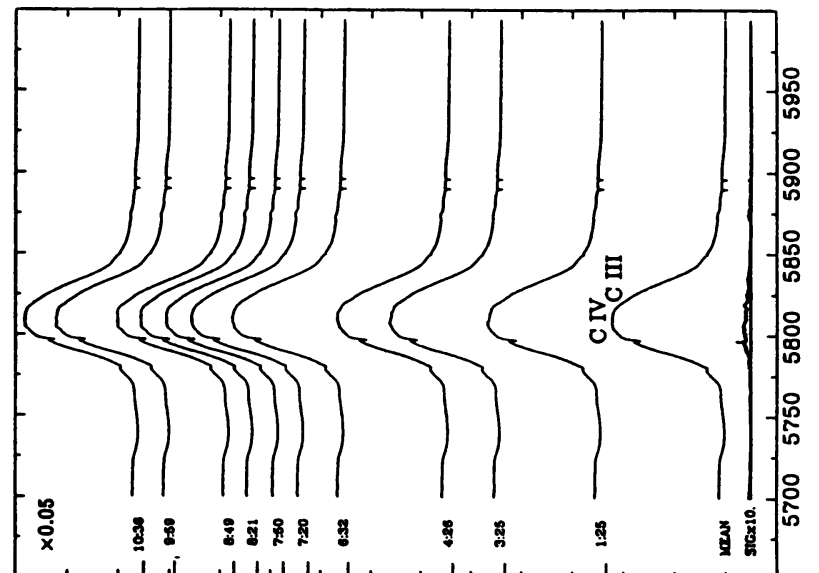

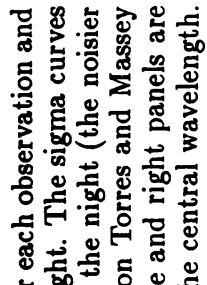

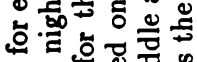

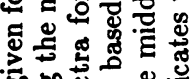
.0 올

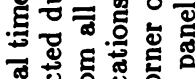

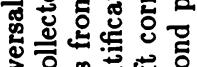

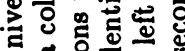

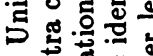
氙.

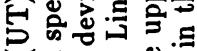

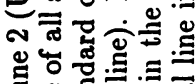
今。 क्ष

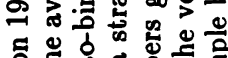
ธี

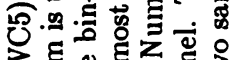
¿छฏ

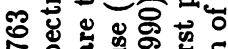

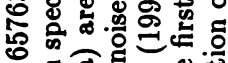

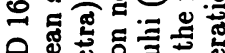

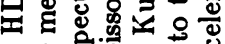
월 के

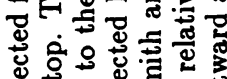

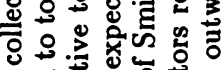

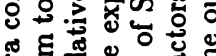

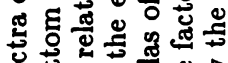

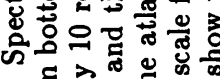
क छี

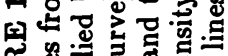

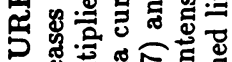
월

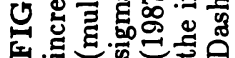

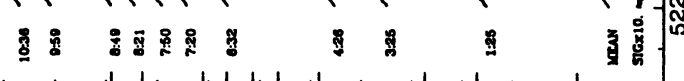

然 


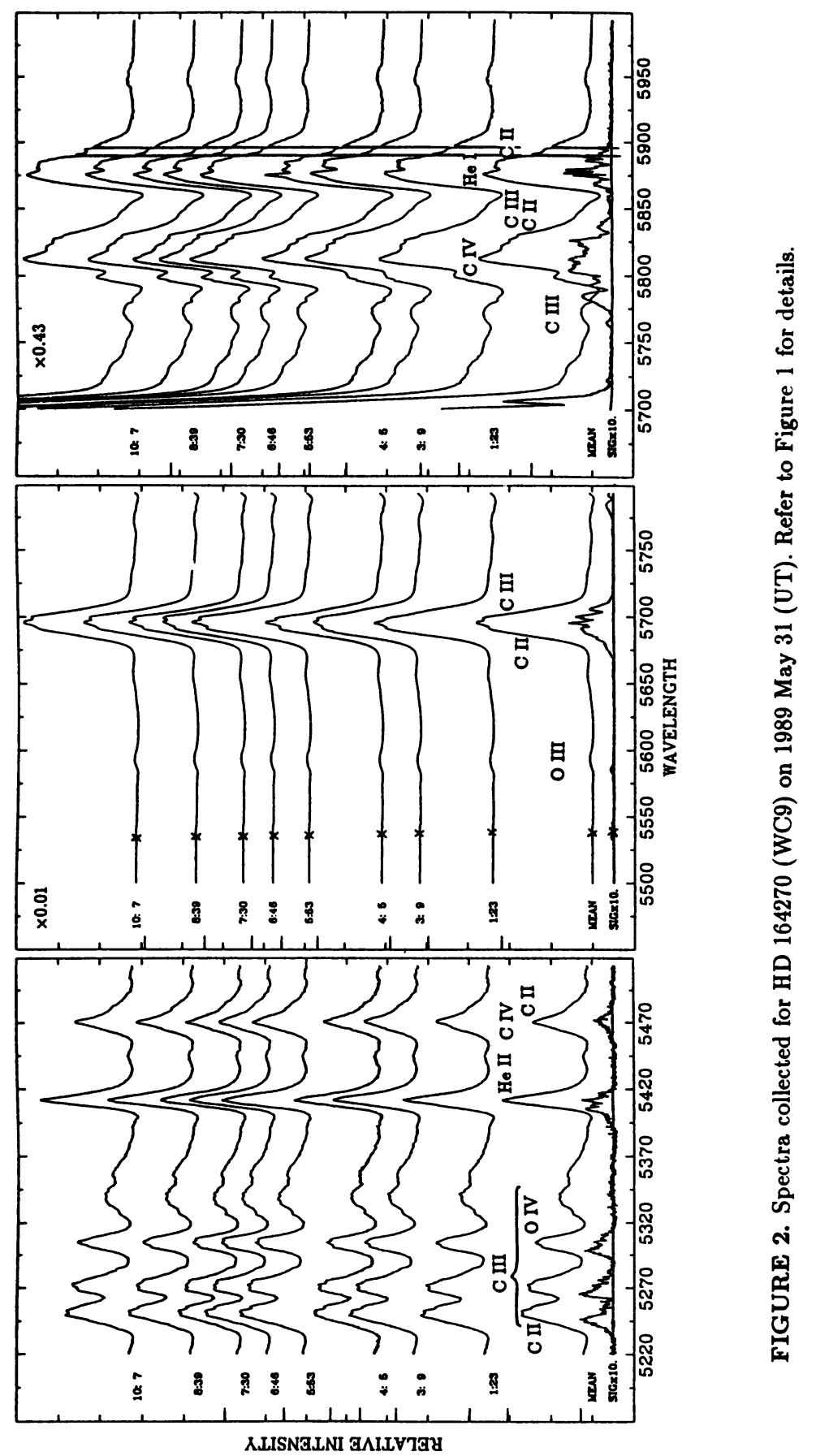




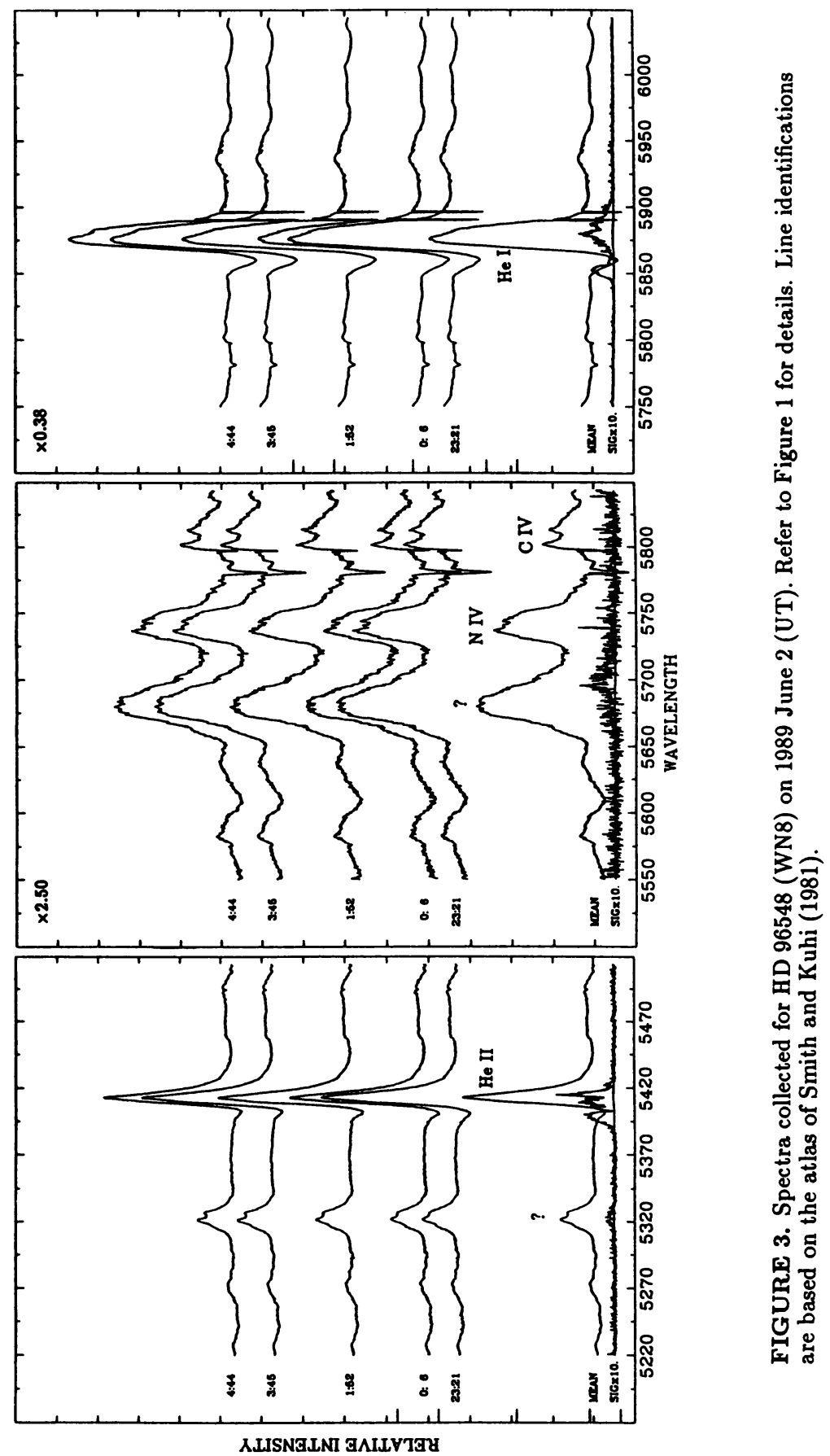


order of $10 \mathrm{hr}$ for most stars observed to date. One can clearly see that bumps that start red-shifted relative to the line center become even more red-shifted with time, while the blue-shifted bumps become more blue-shifted (see other examples in Robert et al. 1988, Robert and Moffat 1989). Figure 4 shows the O III 5592 P Cyg profiles of HD 164270, where one can see moving bumps in emission and dips in absorption (or yet more emission bumps?), both of which are of similar amplitude. These structures are interpreted as a consequence of wind density enhancements or "blobs" moving outwards along with the general wind.

For the three newly observed stars, the same general pattern of bumps is seen in the variable lines at the same time. An example of this behavior for the WC9 star HD 164270 is given in Figure 5. (Further work needs to be done to check for possible time delays between the lines.) In HD 191765 , all the Pickering He II emission lines tend to vary in the same way, while He II 4686 shows some differences and N IV 4058 varies quite differently (McCandliss 1988).

\section{2) Quantifying the inhomogeneities}

Table I gives the average height, maximum height and the average FWHM of the bumps in the most variable lines for each star. The last column indicates the typical number of inhomogeneities identified at any given time. This parameter is more easily deduced (especially for the narrower lines) by subtracting first the overall average spectrum.

\begin{tabular}{|c|c|c|c|c|c|c|c|}
\hline \multirow[t]{15}{*}{ TABLE I } & $\overline{\mathrm{HD}}$ & $\overline{\mathrm{Sp}}$ & Variable lines & \multicolumn{2}{|c|}{$\begin{array}{l}<\text { Height }>\text { Max. Height } \\
\text { (line flux units) }\end{array}$} & $\begin{array}{c}<\text { FWHM }> \\
(\AA)\end{array}$ & $\begin{array}{l}\text { No. of } \\
\text { "blobs" }\end{array}$ \\
\hline & \multirow[t]{2}{*}{96548} & WN8 & He II 5411 (P Cyg) & $5 \%$ & $13 \%$ & 2 & $2-3$ \\
\hline & & & He I 5876 (P Cyg) & $6 \%$ & $14 \%$ & 3 & $2-3$ \\
\hline & \multirow[t]{9}{*}{164270} & WC9 & C III 5250 (blended) & $11 \%$ & $22 \%$ & 2 & 3 \\
\hline & & & C III 5272 & $13 \%$ & $26 \%$ & 2 & 3 \\
\hline & & & C III 5305 (blended) & $14 \%$ & $36 \%$ & 2 & 3 \\
\hline & & & He II 5411 (P Cyg) & $8 \%$ & $23 \%$ & 2 & $3-4$ \\
\hline & & & C IV 5471 (blended) & $11 \%$ & $23 \%$ & - & - \\
\hline & & & O III 5592 (P Cyg) & $15 \%$ & $40 \%$ & 2 & 3 \\
\hline & & & C III 5696 & $10 \%$ & $14 \%$ & 2 & $4-5$ \\
\hline & & & C IV 5805 (blended) & - & - & - & - \\
\hline & & & He I 5876 (blended) & - & - & - & - \\
\hline & \multirow[t]{2}{*}{165763} & WC5 & C III 5696 & $7 \%$ & $15 \%$ & 4 & $8-9$ \\
\hline & & & C IV 5805 & $2 \%$ & $4 \%$ & 4 & 8-9 \\
\hline & 191765 & WN6 & $\begin{array}{l}\text { He II } 5411 \\
\text { (Moffat et al. 1988) }\end{array}$ & $7 \%$ & $14 \%$ & 4 & $4-5$ \\
\hline
\end{tabular}

Early-type stars appear to show a greater number of discrete bumps in their spectral lines at a given time compared to late-type stars. (Here we make no distinction between the different sizes of the blobs. In future work, the mass function of the blobs will be considered.) The inhomogeneities in early-type stars also tend to display a larger spread in velocity (i.e. <FWHM > of the blobs is larger). These same structures are also likely to be responsible for the variations seen in photometry and polarimetry. In the case of early-type, hot WR stars, the combined effect of a larger number of discrete blobs should lead to a lower net amplitude in the photometric or polarimetric variability as is indeed seen for these stars. For the late-type, cooler WR stars, the bumps tend to be less frequent, narrower (and more intense in late WC), suggesting a higher over-density of wind material in their blobs. This could imply stronger asymmetry of the electron distribution around late-type WR stars, which will result in a larger amplitude of the polarimetric variations, as seen. Denser but fewer simultaneous knots in the winds of late-type stars may also explain the greater photometric variations observed in these stars.

\section{CONCLUSION}

The overall evidence so far suggests a common (but unknown) origin for all the non-binary related, intrinsic variations observed in WR stars. Future work will attempt to quantify this statement in a more rigorous way. 


\section{REFERENCES}

Lamontagne, R., and Moffat, A. F. J. 1987, Astron. J., 94, 1008.

McCandliss, S. R. 1988, Ph. D. thesis, University of Colorado.

Moffat, A. F. J., Drissen, L., Lamontagne, R., and Robert, C. 1988, Ap. J., 334, 1038.

Robert, C., and Moffat, A. F. J. 1990, in Properties of Hot Luminous Stars, ed. Garmany (San Fransisco: Astronomical Society of the Pacific), p. 271.

Robert, C., Moffat, A. F. J., Bastien, P., Drissen, L., and St.-Louis, N. 1989, Ap. J., 347, 1034.

Robert, C., Drissen, L., and Moffat, A. F. J. 1988, in Physics of Luminous Blue Variables, ed. Davidson et al. (Dordrecht: Kluwer), p. 299.

Smith, L. F., and Kuhi, L. V. 1990, preprint.

Smith, L. F., and Kuhi, L. V. 1981, J. I. L. A. Report, No. 117.

Torres, A. V., and Massey, P. 1987, Ap. J. Suppl., 65, 483.

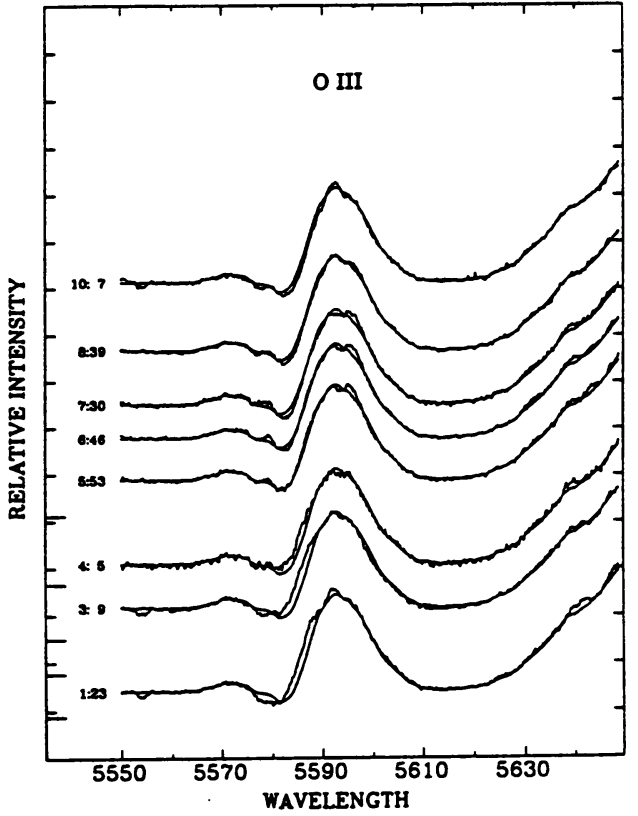

FIGURE 4. P Cyg profiles of the O III 5592 line of HD 164270 on 1989 May 31 (UT). Universal time is given for each observation. The smooth curve superposed on the profiles is the overall mean spectrum for the 3 nights. Bumps and dips are seen on top of the emission and the absorption parts of the profile.

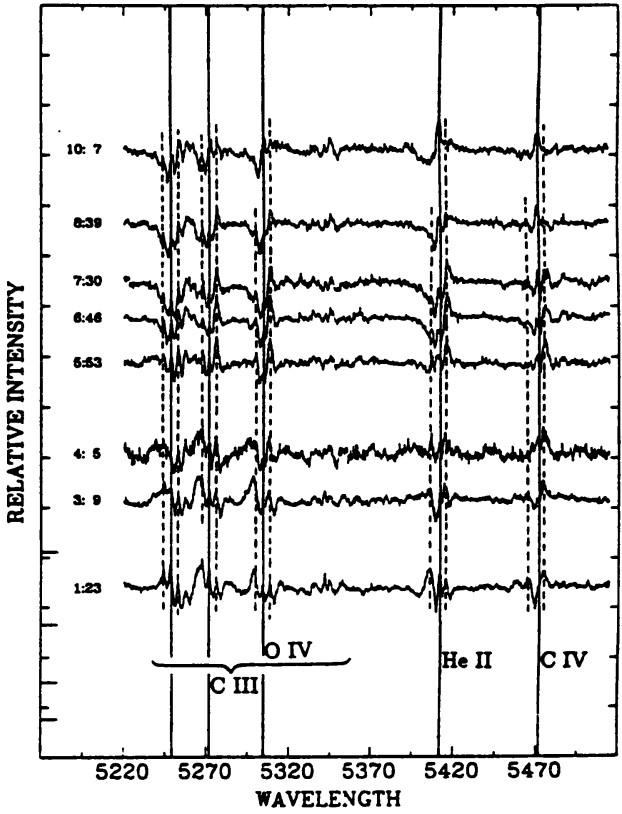

FIGURE 5. Difference from the mean (3 nights) of HD 164270 obtained on 1989 May 31 (UT). Universal time is given for each observation. The vertical solid lines indicate the mean central wavelength for five emission lines shown in Figure 2 (first panel). Dashed lines show the radially outwards acceleration of two sample blobs (one red and one blue relative to the line centers). These two blobs (and others not indicated here) are seen in all lines at the same time in this star. 


\section{DISCUSSION}

Owocki: I found it very surprising that you see simultaneous absorption dips and emission bumps. Do these emission bumps only occur on the blue, or also on the red side of emission features?

Robert: When we see an absorption dip in the absorption part of a P Cyg profile, there is always a blue emission bump at the same time. The emission part of the profile usually shows many blue and red structures at the same time but it is not clear that there is a correlation (at the same speed on each side) between the blue en red bumps (as expected if there was shell ejection).

Cherepashchuk: Could you compare the amplitudes of fine spectral variability for $\mathrm{HeI}$ and HeII lines?

Robert: For WR40, the amplitude seems to be stronger for $\mathrm{HeI}$ lines. There are some maximum heights, there are some structures that are higher but maybe it is too soon to say that there are strong differences between the two lines.

Prinja: In our studies of the $\mathrm{O}$ stars, one of the main things that enabled us to rule out something like a blob model was the very slow velocity laws that were implied by the motions of the single features. Do you have any estimates for the masses of the radially propagating blobs, and - if time-averaged - how this compares with the mass-loss rate of the WR stars?

Robert: No work has been done yet on the mass of the material in the blobs. But we can see already that the range of masses we expect to find will be large. Also, it seems that the number of large blobs is smaller compared to the number of small blobs.

Koenigsberger: Is there any correlation between the frequency of occurrence of the emission spikes and the intensity of the line for a given star?

Robert: The $S / N$ can be significantly small in weak lines compared to the strongest lines. But, as in the case of WR111, even the weakest lines show many bumps that are easy to count. This is a delicate question, and in the future we will need to strictly define the way to identify each bump in order to make good statistics.

de Groot: Supposing these blobs are real, once you have lined them up you can determine acceleration. Did you do this? And are the accelerations variable too?

Robert: There is an example of this type of study in the Proceedings of the BoulderMunich Workshop (Robert and Moffat, 1990). There we compare the blob velocities with the general wind velocity law $\left(v_{\infty}\left(1-R_{*} / r\right)^{\beta}\right)$. This revealed that maybe $\beta>1$. Further work will be done on this topic in the near future. 


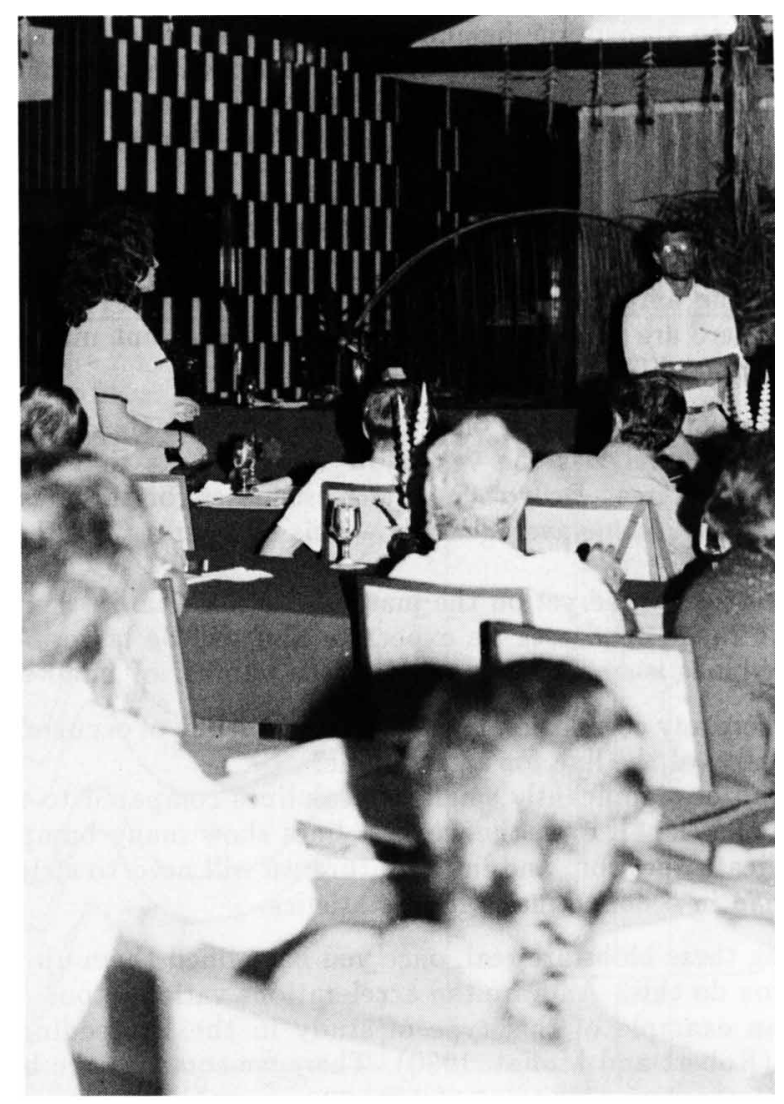

Koenigsberger, Willis chairing 